\title{
Design and Ballistic Performance of Hybrid Composite Laminates
}

\author{
Tomasz K. Ćwik ${ }^{1}$ - Lorenzo Iannucci ${ }^{1}$ - Paul Curtis ${ }^{1,2}$ • \\ Dan Pope ${ }^{1,2}$
}

Received: 16 September 2016 / Accepted: 28 September 2016 / Published online: 15 October 2016

(C) The Author(s) 2016. This article is published with open access at Springerlink.com

\begin{abstract}
This paper presents an initial design assessment of a series of novel, cost-effective, and hybrid composite materials for applications involving high velocity impacts. The proposed hybrid panels were designed in order to investigate various physical phenomenon occurring during high velocity impact on compliant laminates from a previous study on Dyneema® and Spectra®. In the first, screening phase of the study twenty different hybrid composite laminates were impacted with $20 \mathrm{~mm}$ Fragment Simulating Projectiles at $1 \mathrm{~km} / \mathrm{s}$ striking velocity. The best performing concepts were put forward to phase II with other hybrid concepts involving shear thickening fluids, commonly used in low velocity impacts. The results indicated that it is possible to design hybrid laminates of similar ballistic performance as the reference Dyneema® laminate, but with lower material costs. The optimal hybrid concept involves a fibre reinforced Polypropylene front and a Dyneema® backing.
\end{abstract}

Keywords Hybrid · Polymer·Impact · Ballistics

\section{Introduction}

Composite materials have found application in many industries owing to their high specific properties and the ability to tailor them to particular needs. In recent years, however, there has been also a substantial growth in research dedicated to design and evaluation of novel hybrid composite materials. It is believed that the hybridization will allow further optimization of the mechanical performance and that it will also result in a further reduction of the final product weight or increased performance. The latter is of particular importance for defence applications

Lorenzo Iannucci

1.iannucci@imperial.ac.uk

1 Department of Aeronautics, Imperial College of Science and Technology, London, UK

2 DSTL, Porton Down, UK 
as protection systems add a substantial burden to the overall weight of the designed structures. An ideal hybrid protection system would provide both protection and structural capability (i.e. stiffness required for structural design of the vehicle).

A number of research publications have been devoted to evaluation of ballistic performance of hybrid composite materials. Hazell \& Appleby-Thomas [1] investigated ballistic performance of carbon fibre hybrid composites. The authors used Dural and Kevlar for their hybridization. The hybrids were impacted with steel balls at 160-358 m/ s striking velocity. It was found that samples with Kevlar backing performed substantially better than ones with Dural backing. The presence of Kevlar at the front face provided little or no improvement in ballistic properties, whereas sandwiching Kevlar or Dural between the carbon fibre laminates provided inferior ballistic properties when weight was taken into account. Wan et al. [2] identified that Aluminium 2024 - CFRP hybrids provided better protection against hypervelocity impact $(9 \mathrm{~km} / \mathrm{s})$ than each of the two materials separately. However, it was found that the hybrids with Kevlar, $\mathrm{Al} / \mathrm{K} / \mathrm{Al} / \mathrm{K} /$ $\mathrm{Al}$, provided the best performance (the back face velocity of the panel and the impact cone were the lowest). Larsson \& Svensson [3] conducted research on structural lightweight composite materials. Hybrids of carbon fibre laminates with Zylon AS layers and Dyneema ${ }^{\circledR}$ SK66 layers were manufactured and ballistically tested. The trials involved $5.46 \mathrm{~mm}$ diameter Fragment Simulating Projectiles (FSPs) and $6 \mathrm{~mm}$ diameter steel balls which were fired at up to $600 \mathrm{~m} / \mathrm{s}$. The percentage of Dyneema® or Zylon AS in the carbon fibre hybrids varied from 0 to $100 \%$. It was found that the hybrids with $25 \%$ of Zylon or Dyneema® had the best ballistic properties while maintained good compression after impact (CAI) properties. Chen et al. [4] investigated influence of various composite layups on the ballistic performance of woven Dyneema ${ }^{\circledR}$ fabric and unidirectional (UD) Dyneema ${ }^{\circledR}$ laminate hybrid. A $23 \times 23 \mathrm{~cm}^{2}$ panels were subjected to impact of $5.5 \mathrm{~mm}$ diameter cylindrical projectiles fired at $400-500 \mathrm{~m} / \mathrm{s}$ striking velocity. It was found that the hybrid with the largest amount of UD plies at the rear face of the panel and only few woven plies at the front gave the lowest back face deformation. Del Rosso et al. [5, 6] investigated mechanical and ballistic performance of microbraid reinforced polymer composites (mBRPC). Composites reinforced with microbraids of different architectures and made of different materials (Dyneema ${ }^{\circledR}$ and Kevlar ${ }^{\circledR}$ ) were subjected to $7.94 \mathrm{~mm}$ diameter steel ball impact. An improvement of up to $20 \%$ in terms of the ballistic limit was noted, compared to similar laminates reinforced with unidirectional fibres. Fujii et al. [7] conducted a study on ballistic behaviour of CFRP hybrids using $4 \mathrm{~mm}$ diameter steel spheres fired at 500-1230 m/s. Three different types of carbon fibres were used for the study. The authors found that carbon fibres in the front part of the material had very little influence on the impact energy absorption, as the region experienced hydrodynamic behaviour. However, presence of high tenacity fibres at the rear part of another hybrid had positive influence on the ballistic performance of the hybrid. The authors noted that all $2 \mathrm{~mm}$ thick specimens showed similar energy absorption above striking velocities of $711 \mathrm{~m} / \mathrm{s}$ due to the hydrodynamic behaviour. Sevkat [8] evaluated ballistic performance of plain weave S2-glass and IM7 carbon fibre hybrid laminates with toughened SC-79 matrix system, using .22 cal copper bullets. A non-hybrid carbon fibre laminates were used as reference. It was found that the presence of the glass fibre skins increased the $\mathrm{V}_{50}$ ballistic limit of the material by approximately 70 to $317 \mathrm{~m} / \mathrm{s}$. Unfortunately, although there exist a number of publications related to high velocity impacts on monolithic (i.e. non-hybrid) compliant laminates [9-17], only few are related to design of novel hybrid structures. 
The results presented in this paper come from a short study which aimed to identify whether it is possible to improve ballistic performance of commercially available high performance materials by hybridizing them with other materials, and whether it is possible to design hybrid laminates which have the same ballistic performance as the materials available on the market, but substantially lower material costs. The study comprised of two phases: first, the initial screening of various concepts, then a more detailed evaluation of the selected few concepts and addition of few additional concepts.

\section{Experimental Setup}

In phase I of this study, a series of impacts were performed on a number of different hybrid concepts at a specific impact velocity, typically in the region of $1 \mathrm{~km} / \mathrm{s}$. The greatest scatter is usually near the $\mathrm{V}_{50}$ of the system, and thus an objective measure could be used to evaluate the single shot performances. The residual velocity and deformation pattern were examined and the energy dissipated calculated. The reference material was pristine Dyneema® HB26 with a number of hybrids system substantially cheaper than this reference system. The hybrid systems which were substantially better (at least 10-20\%) in terms of the ballistic performance or to be substantially cheaper while maintaining the same ballistic performance as the reference Dyneema® HB26 system.

In phase II of the study the selected concepts were further tested and their ballistic curves were compared with the reference system. Due to the limited amount of materials available, only three to four firings were performed per material type. As the consequence, the presented data is limited and no $\mathrm{V}_{50}$ ballistic limits were derived. The trials were conducted in a similar process to those described in $[18,19]$ with $20 \mathrm{~mm}$ copper Fragment Simulating Projectiles (FSPs) fired by a powder gun at a normal angle to the surface of the panels. The projectiles had exactly the same geometry as the steel projectiles specified in the STANAG 2920 [20], but higher mass (as a consequence of higher mass density of copper) with a weight of $60.25 \pm$ $0.25 \mathrm{~g}$. All panels tested were $250 \times 250 \mathrm{~mm}^{2}$, with an effective impact window of $200 \times 200$ $\mathrm{mm}$, with the perimeter clamped within the overlap of frame. This was determined based on the time required for a wave to reach the edge boundaries and return to the impact site. Ideally the impact event should finish before the wave interacts with the returning wave and potentially influencing the results. The nominal areal density of all panels in phase I and phase II studies were the same as the previous studies [18, 19] i.e. $23.5 \mathrm{~kg} / \mathrm{m}^{2}$, unless indicated otherwise. The same experimental setup was also used. The experiments were captured by a Vision Research high speed cameras, Phantom V711. A typical setup of the cameras is presented in Cwik et al. [19].

\section{Hybrid Design}

The aim of the presented research was to develop a hybrid composite laminate of improved ballistic performance or lower material cost and similar performance as Dyneema ${ }^{\circledR}$ HB26, which was selected as the reference material. An extensive analysis of ballistic performance of Dyneema ${ }^{\circledR}$ HB26 and Spectra 3124 was given by Ćwik et al. [19].

The design of the presented hybrid laminates was largely based on Ćwik et al. findings. The authors noted that the First Major Delamination (FMD), which was created during deformation 
due to projectile impact, seemed to indicate the border between the front part of the panel experiencing inelastic deformation (shearing and hydrodynamic behaviour to a large extent) and the rear part of the panel acting as a membrane catching the projectile. The rear part of the panel dissipated kinetic energy of the projectile via various mechanisms such as fibre straining, shear deformation, and lateral deformation of the panel. Figures 1 and 2 present location of the FMD in Spectra 3124 and Dyneema ${ }^{\circledR}$ HB26 panels, respectively, provided in that study. The location was measured both manually (using a calliper) and based on the high speed videos details are provided in Ćwik et al. [19]. The charts show that the location of the FMD changed with increase of the striking velocity. At low striking velocities, the FMD was located about one third of the panel thickness away from the impact face, while at higher velocities it tended to be located approximately two thirds of the panel thickness away from the striking face. It is noted, however, that the location of the FMD might be, in general, affected by the panel size. It is possible that in larger panels the FMD would be located at different depths than the ones presented in this paper and in [19]. This would be a consequence of having different distance for the longitudinal waves travelling through fibres within the panels during the impact. The interaction between these waves and the transverse waves (though panel thickness) would affect the location of the FMD.

In the present study it was concluded that the front part of the panel does not necessarily need to be of fabricated from an expensive high strength fibre based on the above findings. Therefore, a number of hybrid concepts were designed to validate the importance of First Major Delamination i.e. the location of FMD was used as a guide for design of the hybrids. Also, some of the hybrid designs were inspired by the research described in [21], in which it was found that dry carbon fibre prepreg plies had better ballistic performance than similar cured laminates.

\subsection{Phase I Concepts}

In phase I of the study the different parts of the hybrid panels were adhesively bonded together using Permabond Polyolefin Primer and Permabond 105 adhesive at the edges of the panel. During the hybridisation process seven different materials were used to create 20 different hybrid concepts, which are schematically depicted in Fig. 3 and outlined in Table 1. The

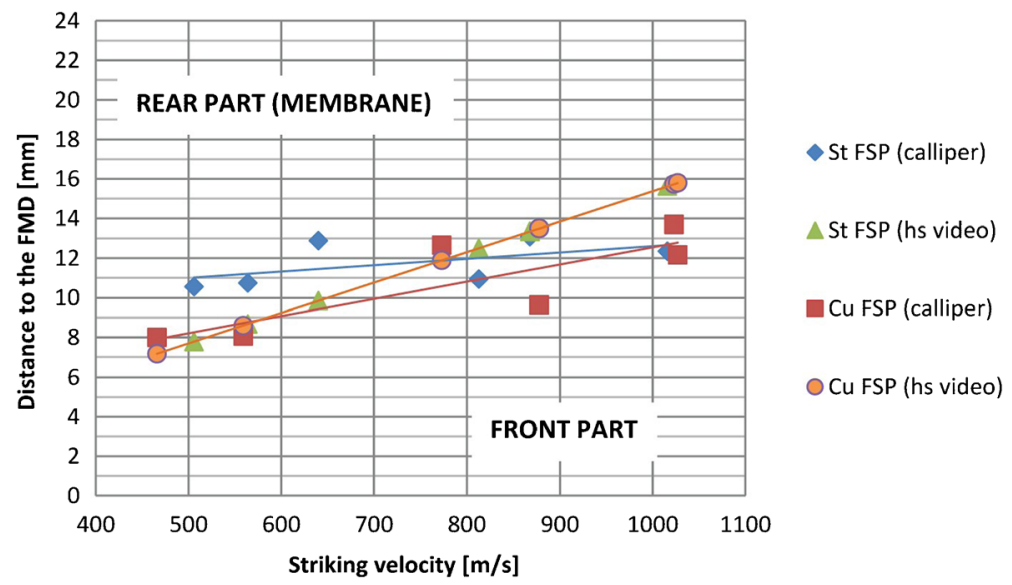

Fig. 1 Location of the FMD for Spectra ${ }^{\circledR} 3124$ panels [19] 


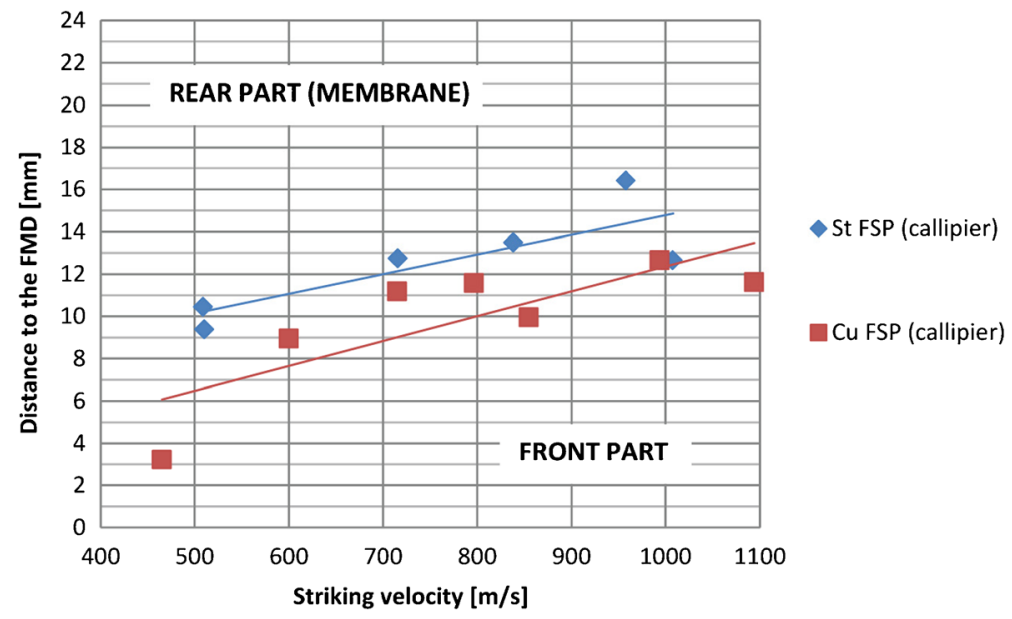

Fig. 2 Location of the FMD for Dyneema ${ }^{\circledR}$ HB26 panels [19]

reference Dyneema® ${ }^{\circledR}$ was used as the backing layer of each hybrid and is depicted as a white rectangle, while other materials are given a specific colour. The different areal weight ratios are indicated in Fig. 3 by the percentage values written in the particular material (colour).

\subsection{Results and Discussion}

The relative ballistic performance comparison between the hybrids was made by normalizing the ballistic performance of a given hybrid with respect to the reference material. The following equations were used:

$$
\begin{aligned}
N B P_{\text {reference }}= & \left(1-\frac{V_{\text {Rref }}}{V_{\text {Sref }}}\right) \times 100 \\
N B P_{\text {material }}= & \frac{\left(1-\frac{V_{\text {Rmat }}}{V_{\text {Smat }}}\right) \times 100}{N B P_{\text {reference }}}
\end{aligned}
$$
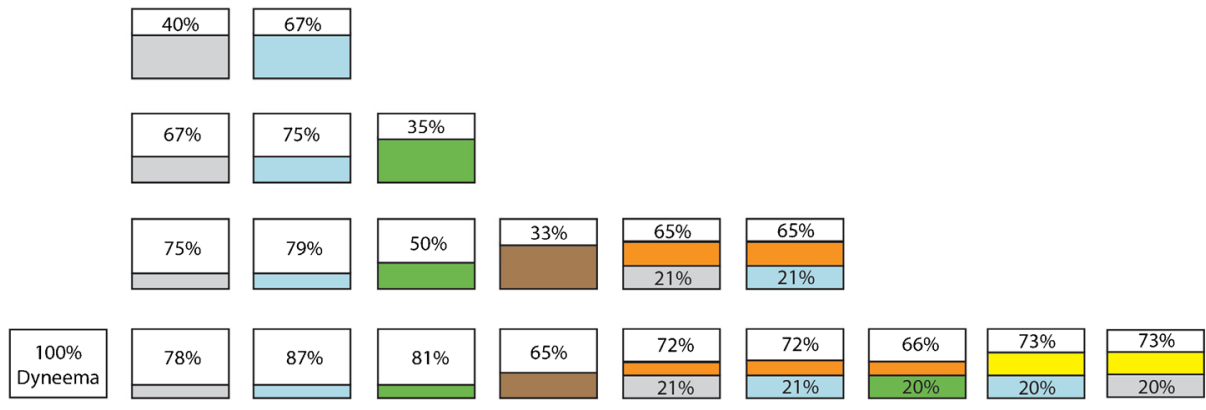

Reference UHMWPE PP Tegris Alu
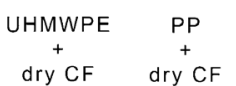

Tegris

dry CF

$\stackrel{+}{\operatorname{dry} C F}$

dry CF

PP

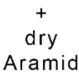

UHMWPE

Aramid Aramid

Fig. 3 Schematic diagram of the hybrid laminate designs investigated in phase I of this study 
Table 1 Phase concepts investigated based on performance at $1 \mathrm{~km} / \mathrm{s} 20 \mathrm{~mm}$ copper FSP

Concept 1: Ultra High Molecular Weight Polyethylene (UHMWPE) + Dyneema®)

Concept 2: Polypropylene (PP) + Dyneema ${ }^{\circledR}$

Objectives

These two concepts have been designed to investigate the maximum amount of front material which can be replaced in a high performance composite. The thickness of the front material was varied and encompassed the reference FMD location. If a large amount of front material is replaced (i.e. beyond the FMD location), there should be a drop in the ballistic performance of the hybrid (i.e. the projectile residual velocity should be higher). For this purpose four different weight ratios were investigated for each concept. It is noted also that the price of each substitute was substantially lower than the price of Dyneema®.

The monolithic UHMWPE $\left(0.97 \mathrm{~g} / \mathrm{cm}^{3}\right)$ has a similar density to Dyneema®, while PP is lower $0.87 \mathrm{~g} / \mathrm{cm}^{3}$.

Concept 3: Tegris + Dyneema ${ }^{\circledR}$

Objectives

This concept is similarly to concepts 1 and 2 and was designed to identify whether a substantial part of the Dyneema ${ }^{\circledR}$ front face could be replaced with a cheaper composite substitute, Tegris. However, compared to a bulk UHMWPE or PP it is commonly used in ballistic applications, but is more expensive than concept 1 and 2, but still cheaper than monolithic Dyneema®. This hybrid concept, which is based on an aligned PP and amorphous PP resin, should give a higher performance compared to the bulk UHMWPE or the PP on the front face of a hybrid material system.

Concept 4: aluminium + Dyneema®

Objectives

The aluminium hybrid was designed to include a material which could have a structural requirement, such as an airframe or automobile structure. In this study a 1050 grade of aluminium was chosen. The isotropic Equation of State $(\mathrm{EoS})$ of aluminium is very different to Dyneema ${ }^{\circledR}$ and to the other aforementioned polymers, Fig. 4, while Table 2 lists the EoS. It is shown that both the sound velocity and the shock velocity in aluminium are much higher than the polymeric materials. Thus, the applied by the projectile shock should reach the backing (i.e. Dyneema $\left.{ }^{\circledR}\right)$ much faster, allowing the material to accommodate the loading quicker than in case of the other polymers and the reference $100 \%$ Dyneema ${ }^{\circledR}$. Figure $4 \mathrm{~b}$ shows that aluminium experiences also much higher shock pressures than the polymeric materials impacted at the same velocity. This implies that the aluminium-projectile interaction might be very different to the polymer-projectile interaction i.e. higher temperatures might be generated at the interface due to shock heating (adiabatic heating). Two hybrids were made: a $35 \%$ aluminium hybrid that had the same areal density as the reference APC, and $66 \%$ aluminium hybrid which had the same thickness as the reference (hence higher mass).

Concept 5: UHMWPE + dry CF + Dyneema $\mathbb{R}$

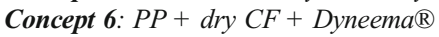

Concept 7: Tegris + dry $C F+$ Dyneema ${ }^{\circledR}$

Objectives

It was observed in Cwik et al. [19] that in some cases there was a localized transition region between the front inelastically deformed part, and the rear membrane part of Dyneema® panel; i.e. the first major delamination was not a step change, but more a transition phase.

The proposed three hybrid concepts were constructed to investigate the possibility to alter the damage mechanisms in this transition area by replacing the middle part of the panel with a fibrous material. A dry carbon fibre (CF) fabric with fibres running at $\pm 45 \mathrm{deg}$. was selected, based on the promising results described in [21].

Concept 8: PP + dry Aramid + Dyneema $\mathbb{R}$

Concept 9: UHMWPE + dry Aramid + Dyneema ${ }^{\circledR}$

Objectives

These two concepts had the same objective as concepts 6,7 , and 8 , but with a higher performance dry aramid fabric.

where NBP is defined as the Normalized Ballistic Performance, and $V_{S}$ and $V_{R}$ denote the striking and the residual velocity, respectively. Figure 5 presents the ballistic comparison. An abbreviation of APC (Aligned Polymeric Composite) was used for Dyneema®.

The UHMWPE hybrids and the PP hybrids indicated that replacement of up to $33 \%$ of the front part of APC with lower cost polymers resulted in hybrids of similar or improved ballistic 
Table 2 Equation of state of the selected materials

\begin{tabular}{lll}
\hline Material & Equation of state & Reference \\
\hline Dyneema® & $1.81+2.72 \mathrm{u}_{\mathrm{p}}$ & {$[22]$} \\
aluminum & $5.39+1.34 \mathrm{u}_{\mathrm{p}}$ & {$[23]$} \\
$\mathrm{PP}$ & $2.9+1.16 \mathrm{u}_{\mathrm{p}}$ & {$[24]$} \\
$\mathrm{PE}$ & $2.34+2.57 \mathrm{u}_{\mathrm{p}}$ & {$[24]$} \\
\hline
\end{tabular}

performance (up to $16 \%$ improvement). However, it is observed that the $22 \%$ UHMWPE hybrid and the $13 \%$ PP hybrid had slightly worse ballistic performance than the reference material. The $19 \%$ Tegris hybrid had almost $60 \%$ better NBP than the reference panel - this hybrid had the best ballistic performance in this part of the study. By replacing $60 \%$ of Dyneema® with UHMWPE, a $30 \%$ worse ballistic performance of the hybrid was obtained. Similarly, the replacement of a substantial part of Dyneema ${ }^{\circledR}$ with Tegris $(50 \%$ and $65 \%)$ gave also hybrids characterized by a worse ballistic performance - $24 \%$ and $28 \%$, respectively. This results appear to support the prediction given by location of the First Major Delamination in Dyneema ${ }^{\circ}$, given in Fig. $1 \mathrm{~b}$ - i.e. at $1 \mathrm{~km} / \mathrm{s}$ striking velocity one should not replace more than approximately $40 \%$ of the front part of the panel (the FMD was located at $10 \mathrm{~mm}$ depth of a $24 \mathrm{~mm}$ thick panel, for the copper FSP).

The $35 \%$ aluminium hybrid showed slightly better NBP than the reference. Although the gain was not substantial, the result indicated that it should be possible to design an aluminiumDyneema ${ }^{\circledR}$ hybrid with almost the same ballistic performance as Dyneema ${ }^{\circledR}$, but with a certain structural design constraint. The $67 \%$ aluminium hybrid, which had the same thickness (not the areal density) as Dyneema®, had slightly worse NBP than the reference Dyneema®.

The insertion of dry carbon fibre fabric between the backing and the UHMWPE (or PP or Tegris) front, resulted in hybrids of substantially worse ballistic performance (20-25\%) than the reference material. The UHMWPE $(21 \%)+$ dry CF $(7 \%)+$ Dyneema ${ }^{\circledR}$ hybrid noted slightly improved ballistic properties, however, more tests should be conducted to confirm this result. Figure 6 shows the signature of the dry carbon fibre on the Dyneema® backing. It appears that the carbon fibres failed in a brittle manner. However, the $20 \%$ PP + $7 \%$ dry aramid hybrid showed an $18 \%$ improvement with respect to the reference. The dry aramid was torn and substantially distorted, but not pulverized. The Dyneema backing bulged outwards, Fig. 7, compared to Dyneema in the hybrid with dry carbon fibres. The hybrid with the UHMWPE front and the dry aramid insert showed marginally worse ballistic performance than the Dyneema®.

a)

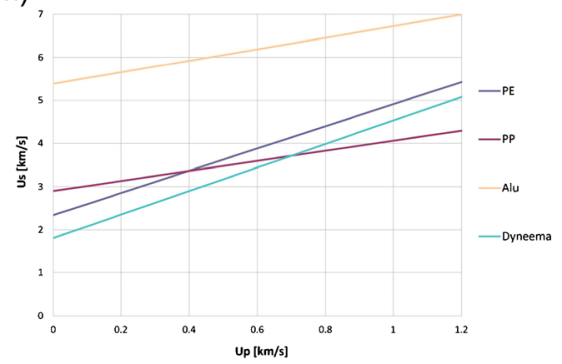

b)

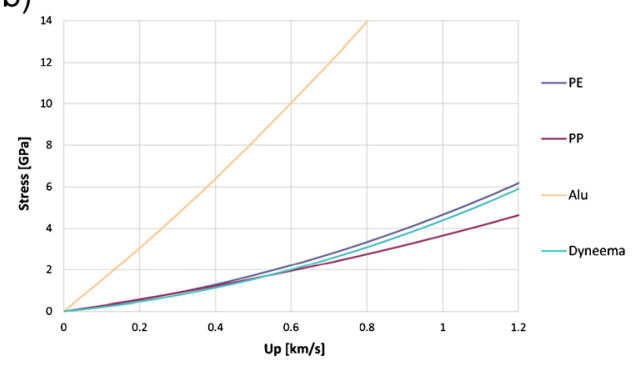

Fig. 4 Equation of state for PE, PP, aluminum, and Dyneema ${ }^{\circledR}$ in the: a $U_{p}-U_{s}$ plane; $\mathbf{b} U_{p}-\sigma$ plane 


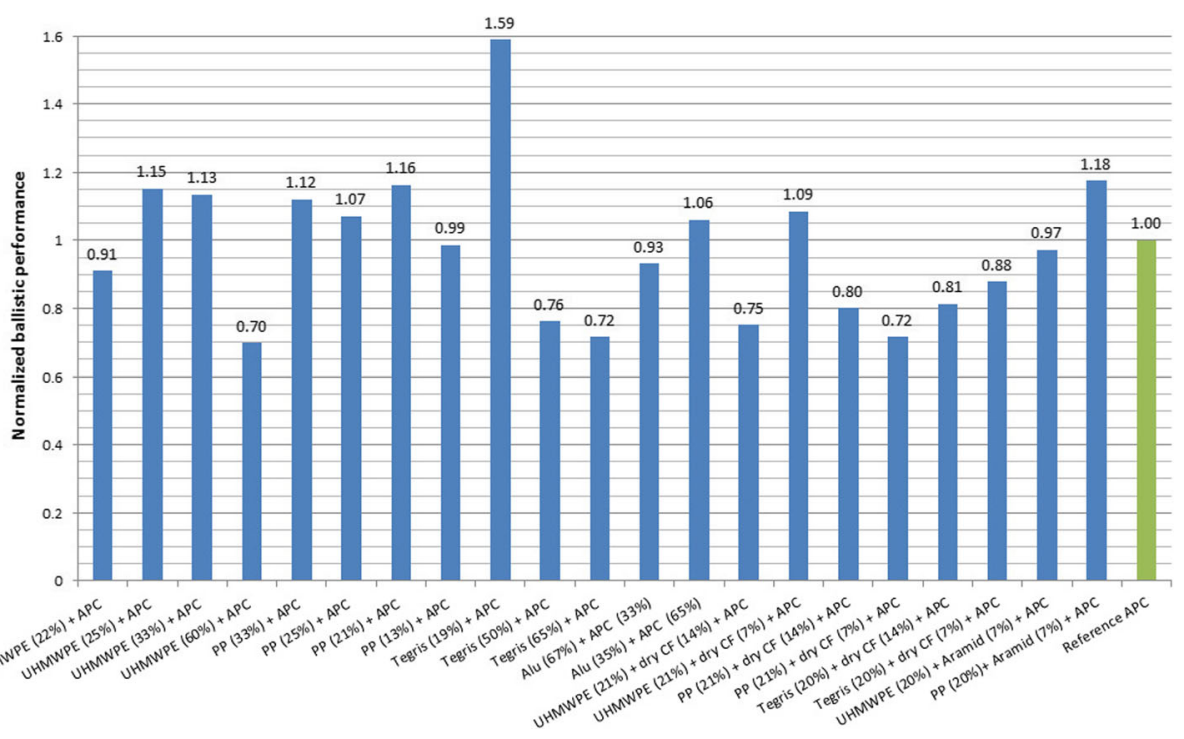

Fig. 5 Normalized ballistic performance of the hybrid panels

\subsubsection{Front Face Deformation}

The analysis of the high speed videos showed that different panel fronts reacted in a different manner to the projectile impact. Figure 8 shows snapshots from high speed videos for four different hybrids. The flash at the initial contact between the panel and the projectile was observed during all experiments. The first column presents deformation in time of the reference Dyneema ${ }^{\circledR}$ panel. A detailed description of front face deformation of Dyneema ${ }^{\circledR}$ was given in [19]. The UHMWPE hybrid (shown in the second column in Fig. 8), on impact, ejected a certain amount of material from the impact area. Hybrids with polypropylene front face initially experienced a similar deformation as the UHMWPE fronts (ejecta etc.) but after a short time interval multiple cracks propagated away from the impact point, resulting in a total disintegration of the PP front part.

Fig. 6 A photo of the inner surface of Dyneema ${ }^{\circledR}$ that was in contact with the dry $\mathrm{CF}$

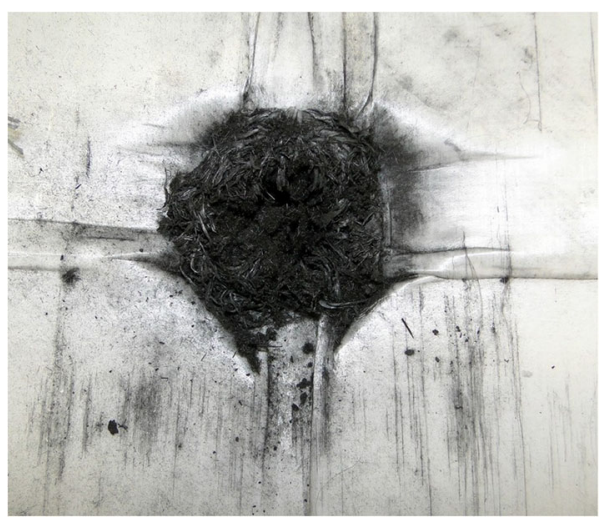


Fig. 7 A photo of the aramidDyneema ${ }^{\circledR}$ interface

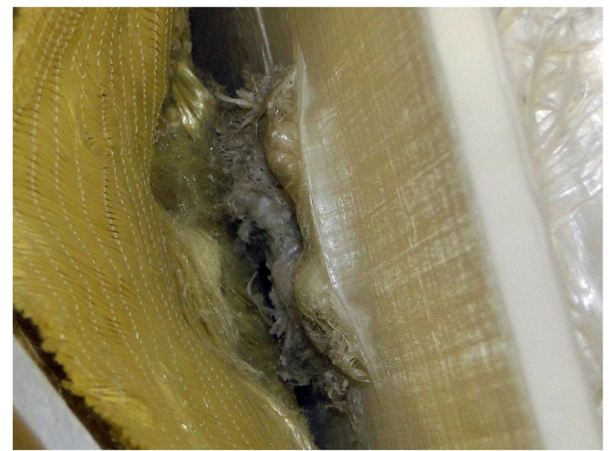

\subsubsection{Back Face Deformation}

The high speed videos of rear face of the tested panels also showed different hybrids deformed differently during the impact event. Examples of the back face deformation are presented in Fig. 9. The figure shows an instance of deformation which took place approximately $60 \mu \mathrm{s}$ after the initial contact between the projectile and the panel front surface. The reference Dyneema ${ }^{\circledR}$ had a diamond-shape deformation (see Fig. 9a). The debonded vertically running primary yarns indicated that the projectile was about to completely penetrate the panel at that instance of time. The deformation of UHMWPE $(25 \%)+$ Dyneema ${ }^{\circledR}$ hybrid were more eye shaped. It appears that the area involved in the deformation was greater than the deformation

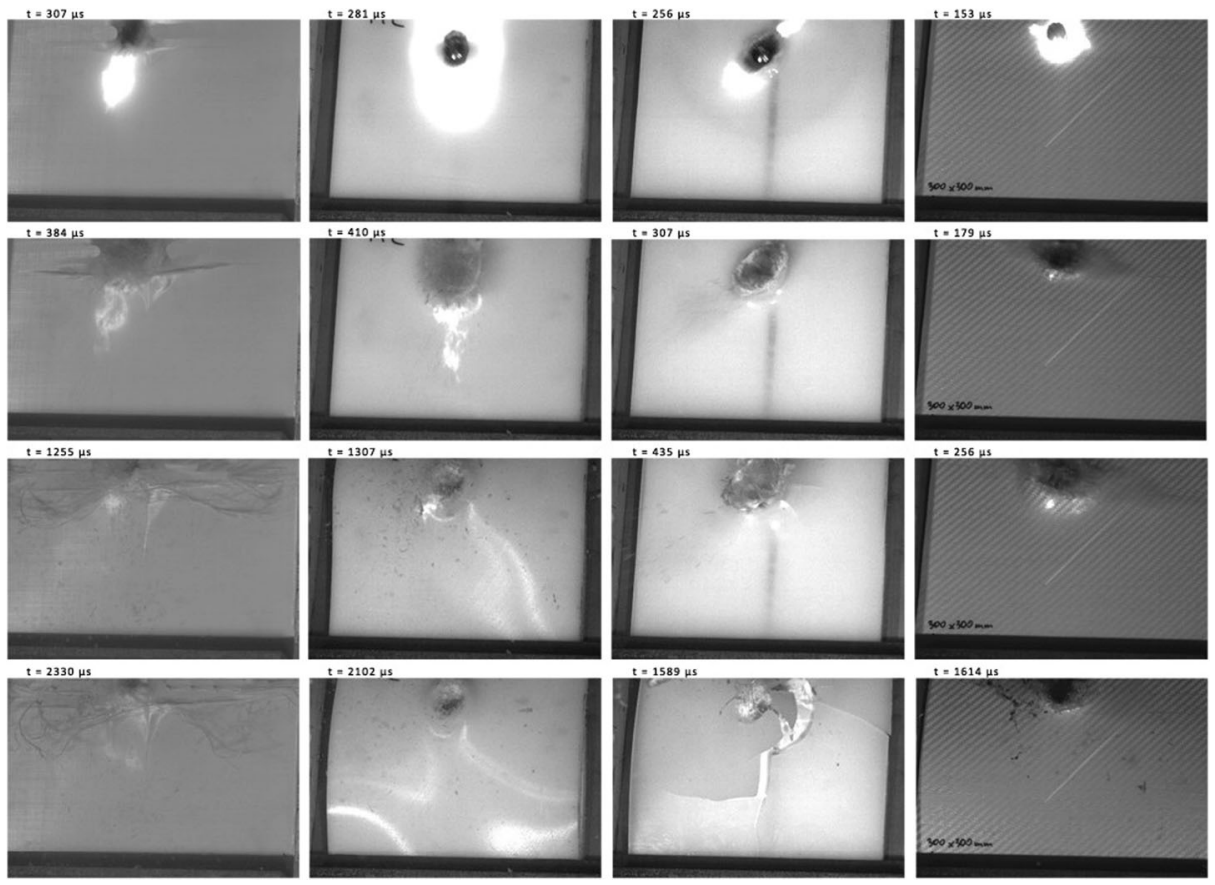

Fig. 8 Snapshots of the front face deformation of different panels during impact. In columns, from left: $100 \%$ Dyneema®, UHMWPE, PP, Tegris 
a)

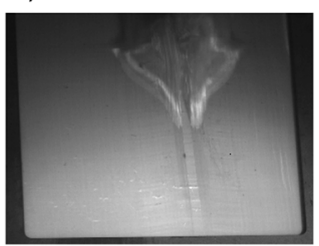

d)

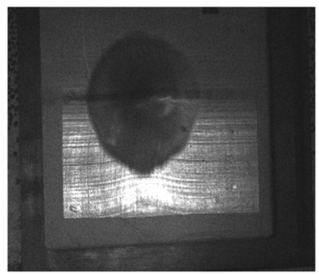

b)

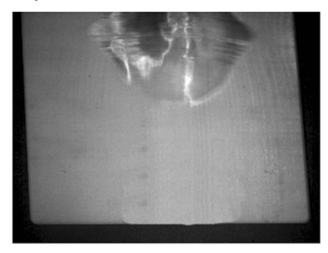

e)

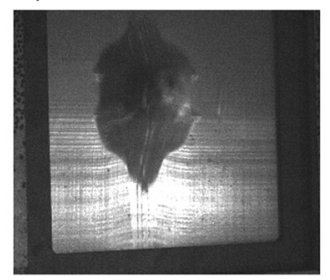

c)

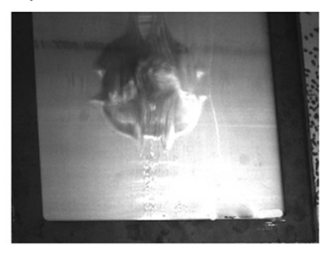

Fig. 9 Snapshots of the back face deformation of: a Reference Dyneema®; b UHMWPE (25\%)+Dyneema®; c PP $(21 \%)+$ dry CF $(7 \%)+$ Dyneema ${ }^{\circledR}$; d Tegris $(19 \%)+$ Dyneema ${ }^{\circledR}$; e Tegris $(60 \%)+$ Dyneema ${ }^{\circledR}$

area of $100 \%$ Dyneema ${ }^{\circledR}$. Also, the extent of the back face drawing in throughout the entire experiment (not shown in here) was greater for the UHMWPE hybrid. The PP (21 \%)+dry CF (7 \%)+Dyneema® hybrid, which noted the worst NBP, was characterized by a circular in shape deformation. A similar type of deformation was observed for the PP (21\%)+dry Aramid $(7 \%)+$ Dyneema ${ }^{\circledR}$ hybrid which had $18 \%$ better NBP than the reference. At the measured instance of time, the panel was just about to be penetrated by the projectile and the deformed area appeared to be of similar size as the deformation of the reference material. A circular shape deformation was observed also for the Tegris hybrids.

\subsubsection{Projectile Deformation}

The projectiles that penetrated the panels were caught by a catcher box; as discussed by Ćwik et al. [19]. It was found that the copper projectiles fired at low velocities (e.g. $200-400 \mathrm{~m} / \mathrm{s}$ ) did not deform during the catching process. However, the projectiles fired at high velocities (e.g. $1 \mathrm{~km} / \mathrm{s}$ ) deformed to a certain extent. Figure 10a shows a projectile that was fired at $1 \mathrm{~km} / \mathrm{s}$ into the catcher box without any panel in the rig. Therefore, the deformation of the projectiles presented in the Fig. $10 \mathrm{~b}, \mathrm{c}$, and $\mathrm{d}$ is a resultant of the deformation during penetration of the tested panel and during the penetration of the catcher box. A typical deformation of a $20 \mathrm{~mm}$ copper FSP which impacted the reference Dyneema ${ }^{\circledR}$ is shown in Fig. 10b. The projectiles that impacted hybrids with the UHMWPE fronts to deform to greater extent than the projectile that impacted the reference material.

\subsection{Summary of Phase I and Phase II Concepts}

\subsubsection{Phase I}

Phase I results indicated that it was possible to achieve improvement of a hybrid panel ballistic performance by replacing in a front part of a Dyneema ${ }^{\circledR}$ panel with other systems, some may be of a lower cost. The type of system used in the front panel affected how the backing 
a)

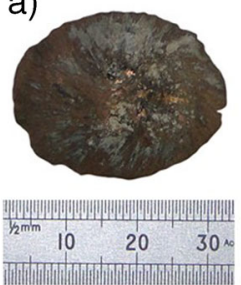

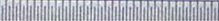
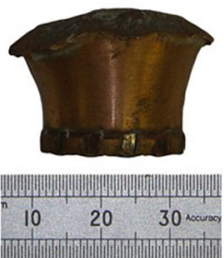

b)
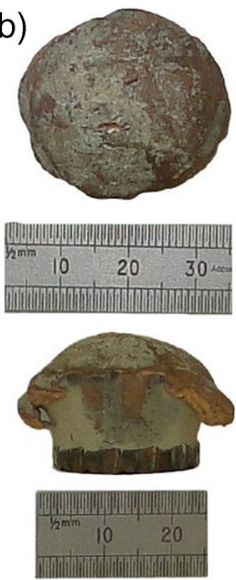

c)
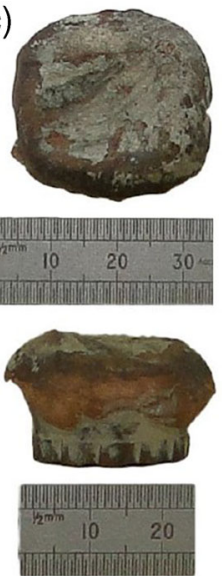

d)
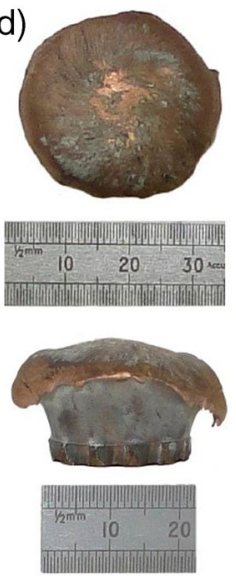

Fig. 10 Images of the deformed copper projectiles that impacted a catcher box with sand; b $100 \%$ Dyneema®; c UHMWPE (25\%)+Dyneema®; d PP (20\%)+Aramid (7\%)+Dyneema®

Dyneema ${ }^{\circledR}$ deformed and alternated deformation of the projectiles. It was generally observed that replacement of excessive amount of Dyneema ${ }^{\circledR}$ produced a worse ballistic performance than the reference $100 \%$ Dyneema®. The results indicated that a bulk UHMWPE was a good low cost candidate for a hybrid front panel. A similar observation was made for polypropylene front panels. The best Normalized Ballistic Performance was obtained by a hybrid system with $19 \%$ Tegris. This was believe to be associated with the fibre reinforcement of the amorphous PP, thus increasing the compressive strength of the PP system.

The results indicate that it should be also possible to design an aluminium-Dyneema ${ }^{\circledR}$ hybrid possessing the same or better ballistic performance than $100 \%$ Dyneema ${ }^{\circledR}$. The inclusion of dry carbon fibre layers in between the front part of hybrid panel and the Dyneema ${ }^{\circledR}$ backing deteriorated the ballistic performance of the hybrid. Nevertheless, a similar hybrid, but with dry aramid fabric showed more promising results.

\subsubsection{Phase II}

It was determined that the amount of material replaced was approximately given by the location of the First Major Delamination obtained from the reference Dyneema ${ }^{\circledR}$ tests. This procedure was also followed in Phase II of the study, in which only three concepts from phase I were investigated in more detail and another four new concepts were evaluated principally based on shear thickening fluid (STF) materials at the front or as the middle layer of the hybrid panels. The hybrid designs and the percentage areal weight ratios of the hybrids constituents are shown in Fig. 11. All panels had nearly the same areal density as the phase I panels and the

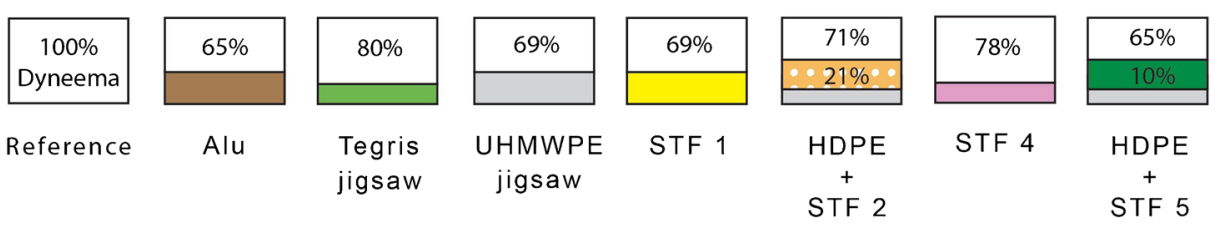

Fig. 11 Schematic diagram of the hybrid laminate designs investigated in phase II 
amount of the replaced front part of Dyneema® ${ }^{\circledR}$ was less than $35 \%$. Four hybrids with shear thickening fluids were investigated based on the following key assumptions;

a) the STFs had slightly lower mass density than Dyneema ${ }^{\circledR}$ or UHMWPE. Hence their hydrodynamic behaviour should be similar,

b) in certain low velocity impact scenarios STFs have been used in the front region of a system and have proved beneficial, e.g. protective motorcycle impact clothing;

c) are of lower cost than Dyneema ${ }^{\circledR}$.

Figure 12b shows a Tegris jigsaw hybrid which was manufactured using a waterjet cutter. Such a system localised damage and potentially could provide a multi-hit capability. Figure $12 \mathrm{~d}$ and e show hybrids with two shear thickening fluids of different architectures. The former, in addition to its strain rate dependence capability, was also an auxetic material (i.e. on through thickness compression the inner parts of the polymer move towards the impact point); while the latter had similar energy absorption capability determined in low velocity impact while lower weight. The shear thickening fluid in STF 4 hybrid, schematically presented in Fig. 11, had similar architecture to STF 2 but larger holes.

\subsection{Results and Discussion}

Figure 13 shows the results of the phase II ballistic tests and the Dyneema® ballistic curve from Ćwik et al. [19]. Due to limitations in the amount of material available for construction of the hybrids, only a limited number of panels (four or less) were tested per hybrid system. As a consequence no ballistic limits were determined. However, the data was sufficient for a rough comparison of the materials performance. The ballistic curve shows that the back face measured residual velocity for firings measured at approximately $750 \mathrm{~m} / \mathrm{s}$ was approximately the same for all panels, however, for firings at $850 \mathrm{~m} / \mathrm{s}$ some of the hybrids behaved differently. Figure 14 shows that, although at the initial stages of the experiments the back face

a)

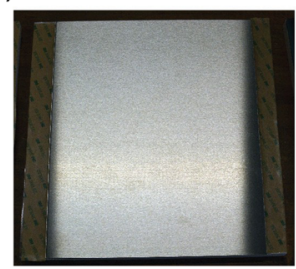

d)

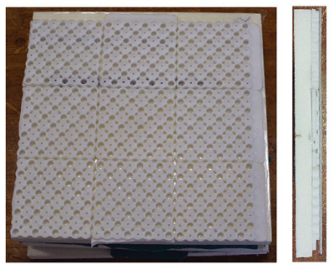

b)

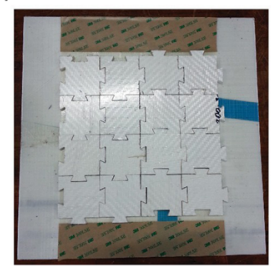

c)

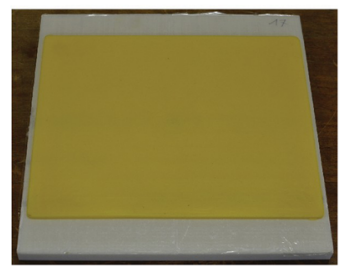

e)

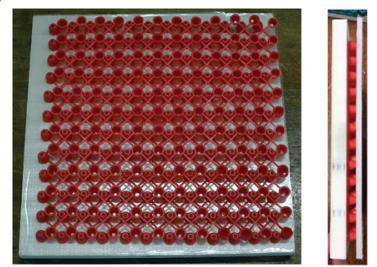

Fig. 12 Photos of the entire panels: a Alu hybrid; b Tegris jigsaw hybrid; c STF 1 hybrid; d HDPE+STF 2 hybrid - front view (without front HDPE layer) and side view of the full panel; e HDPE+STF 5 hybrid - front view (without front HDPE layer) and side view of the full panel 
deformation looked similar for all investigated hybrid systems, both the aluminium hybrid and the STF 1 hybrid were penetrated by the projectile after $180 \mu$ s. The Tegris jigsaw hybrid (Fig. 14a) and the UHMWPE jigsaw hybrid (not shown here as the deformation was very similar to the Tegris system) were not fully penetrated and similar to the aforementioned lower striking velocity impacts. The projectiles via a simple momentum exchange detached the rear part of the panel which travelled with the projectile on exit. The latter was accompanied by creation of a large number of delaminations between the detached part and the remaining panel part in the test rig (the latter consisted of the replacement front and part of Dyneema® material). It was also observed that after the initial 70-80 $\mu$ s the projectile was close to penetrating the aluminium and STF 1 panels. At this point, the primary yarns at the outer surface of the back face and the adjacent secondary yarns started to debond. Soon after the projectile penetrated the very outer layers of the hybrids. Such behaviour was not observed for the Tegris and UHMWPE jigsaw hybrids - the initial impact cone increased in size to the point where the deformation reached the edges of the panel and the part of Dyneema ${ }^{\circledR}$ in front of the projectile completely debonded. These velocities are much lower than the aluminium and STF hybrids (see Fig. 13). Nevertheless, the authors believe that these panels would still show lower residual velocities, if larger panels were used (sufficient to neglect the edge effects), due to the fact that these experiments showed that the back face deformation characteristics were alternated by the selected front panel materials. The remaining two shear thickening fluid hybrids did not affect the performance of the hybrids significantly.

In the phase I of this study it was observed that, on impact, an in-plane bending wave propagated away from the impact point on the front face of the Tegris hybrid. Figure 15c shows that the wave was successfully arrested by the jigsaw construction in the Tegris panel and only the impacted puzzles were damaged. This fulfilled the authors' initial expectations towards the anticipated improvement of multi-hit capability of such front face construction. It remains to be investigated what is the optimal size of such jugsaw puzzle for the best ballistic performance of the panel.

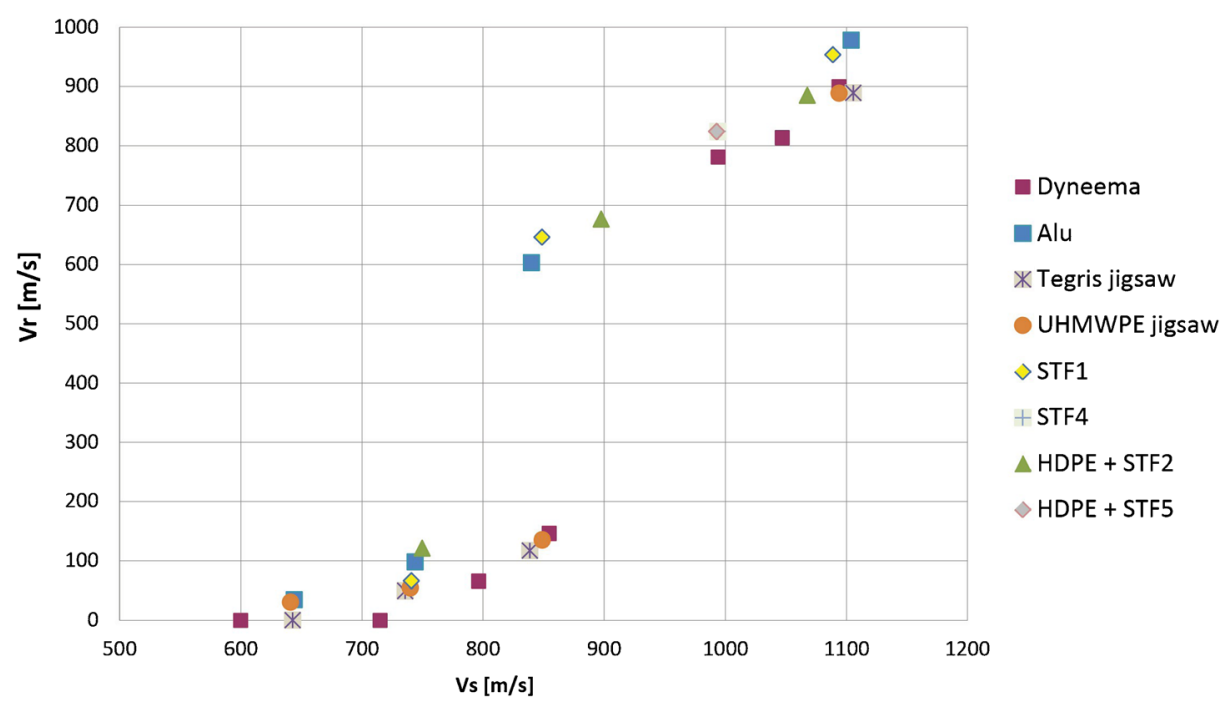

Fig. 13 The ballistic curves of the hybrids investigated in phase II 
Fig. 14 High speed video snapshots of the deformation of the selected hybrids (side view): a Tegris jigsaw hybrid impacted at $839 \mathrm{~m} / \mathrm{s}$; b Aluminum hybrid impacted at $840 \mathrm{~m} / \mathrm{s}$; c STF 1 hybrid impacted at $849 \mathrm{~m} / \mathrm{s}$ a)

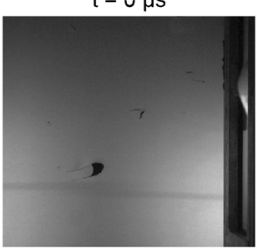

$t=80 \mu s$

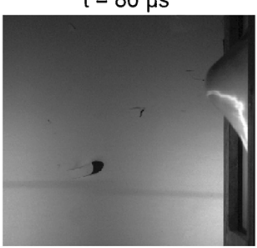

$\mathrm{t}=180 \mu \mathrm{s}$

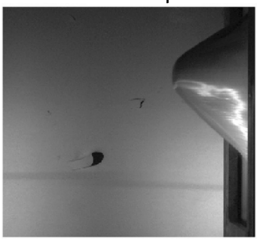

$t=600 \mu s$

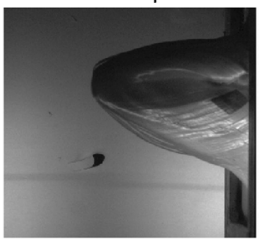

b)

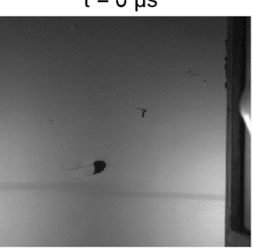

$t=80 \mu s$

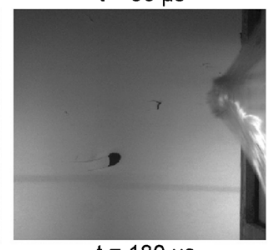

$t=180 \mu s$

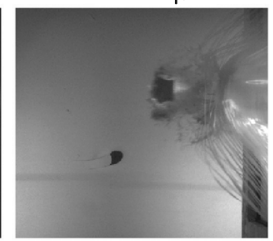

$t=600 \mu s$

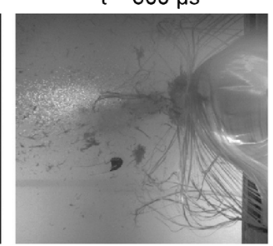

C) $t=0 \mu \mathrm{s}$

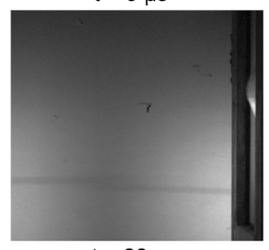

$t=80 \mu s$

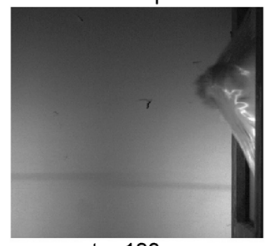

$t=180 \mu s$

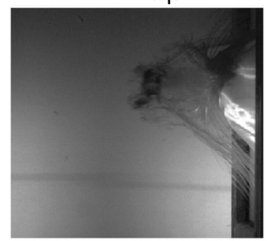

$t=600 \mu s$

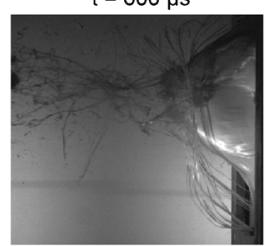

It is worth highlighting that all the observed phenomena played a crucial, from the ballistic performance point of view, role only in the striking velocity range close to the ballistic limit. Figure 13 shows that above $900 \mathrm{~m} / \mathrm{s}$ striking velocity, regardless of what front was used, all residual velocities lied nearly on one single straight line. This implies that at sufficiently high impact velocities/pressures neither the front face material nor the amount of high performance backing material made any difference (mind that in the investigated hybrids different amounts of Dyneema ${ }^{\circledR}$ backing were used, as shown in Fig. 11). Potentially, this might have been a consequence of the inability of the impacted panel to respond to the applied loads before the projectile had locally penetrated the material.

\section{Conclusions}

In summary, it was shown that hybridization of expensive, high performance composite materials with lower cost materials may produce hybrids of improved ballistic performance and reduced material costs. The presented results and conclusions, however, are valid for the investigated threat. The hybrids could respond differently when subjected to a different threat.

The presence of different front materials change the deformation mechanism in the rear Dyneema ${ }^{\circledR}$ part of the panel and the projectiles deformation. The phase I screening tests 
a)

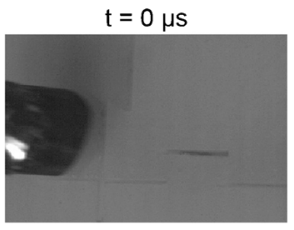

$t=20 \mu s$

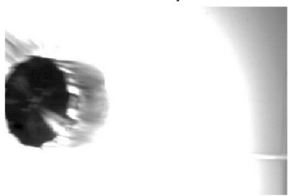

$\mathrm{t}=40 \mu \mathrm{s}$

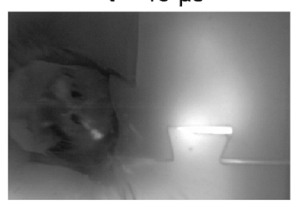

$t=320 \mu s$

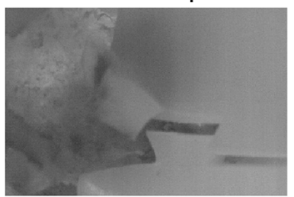

b)

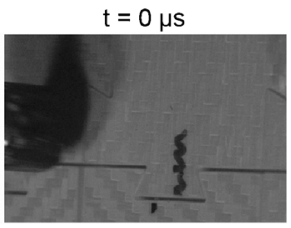

$t=20 \mu s$

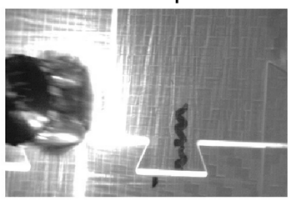

$t=40 \mu s$

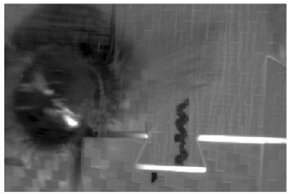

$t=600 \mu s$

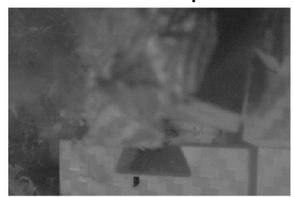

c)

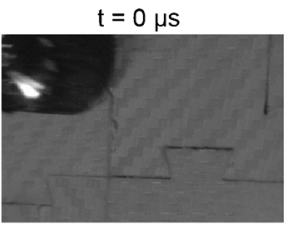

$t=20 \mu s$

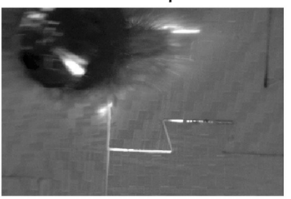

$t=40 \mu s$

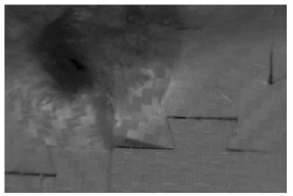

$t=240 \mu s$

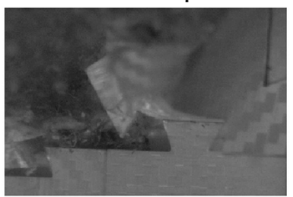

d)

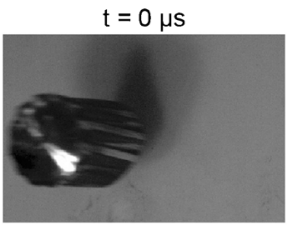

$t=20 \mu \mathrm{s}$

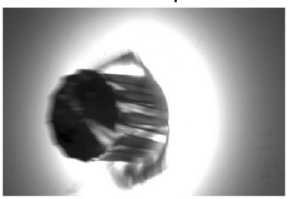

$t=60 \mu s$

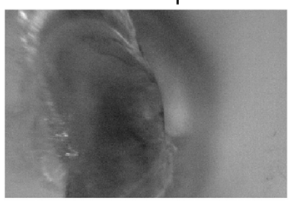

$t=180 \mu s$

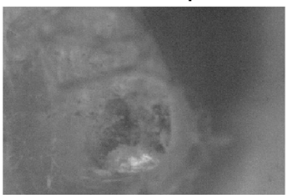

Fig. 15 High speed video snapshots of the front face deformation of the selected hybrids: a UHMWPE jigsaw hybrid impacted at $740 \mathrm{~m} / \mathrm{s}$; b Tegris jigsaw hybrid impacted at $736 \mathrm{~m} / \mathrm{s}$; c Tegris jigsaw hybrid impacted at $839 \mathrm{~m} / \mathrm{s} ;$ d STF 1 hybrid impacted at $741 \mathrm{~m} / \mathrm{s}$

allowed for a quick relative comparison of various concepts and proved the importance of the First Major Delamination. It was shown that the location of FMD obtained from ballistic experiments on $100 \%$ Dyneema ${ }^{\circledR}$ provided a very good indication on how much material at the front of the panel could be replaced. The phase II experiments showed that the four hybrids (aluminium, Tegris jigsaw, UHMWPE jigsaw, and STF 1) provided a similar ballistic performance against the investigated threat as the reference Dyneema ${ }^{\circledR}$, while being of a lower material cost (manufacturing cost was not taken into consideration). Additionally, the jigsaw puzzle structure proved to limit the extent of damage at the front face to the few impacted elements.

The authors believe that further research is required in order to precisely evaluate how much of the expensive high performance material should be replaced with specific substitutes, in order to obtain a hybrid of improved ballistic performance against a given threat at minimum cost. Also, the understanding of importance of various parameters, such as wave speeds, thermal properties, fibre strength, need to be deepened. The $\mathrm{V}_{50}$ ballistic limit should be obtained for each of the hybrids, during trials employing larger panels in order to rule out the influence of edge effects. Also, Digital Image Correlation should be implemented in such trials in order to provide more quantitative data for validation of numerical models [25]. Such numerical models may be able to virtually investigate the design space and predict solutions which may not be obvious. 
Acknowledgments The authors would like to acknowledge support from the EPSRC and the Dstl grant EP/ G042861/1. The support of DSM for providing Dyneema® HB26 material for the phase II of the study is greatly appreciated. The study would not be possible without support from Vision Research UK represented by $\mathrm{Mr}$ Jolyon Cleaves. Special thanks to Dr Adam Connolly, Dr Stefano Del Rosso, Dr Lucio Raimondo, Mr Joseph Meggyesi and Ms Michelle Willows for help during the trials. The shear thickening fluids were donated by $\mathrm{Mr}$ Dan Plant. Dyneema ${ }^{\circledR}$ is a trademark of DSM. Use of this trademark is prohibited unless strictly authorized.

Open Access This article is distributed under the terms of the Creative Commons Attribution 4.0 International License (http://creativecommons.org/licenses/by/4.0/), which permits unrestricted use, distribution, and reproduction in any medium, provided you give appropriate credit to the original author(s) and the source, provide a link to the Creative Commons license, and indicate if changes were made.

\section{References}

1. Hazell, P.J., Appleby-Thomas, G.: A study on the energy dissipation of several different CFRP-based targets completely penetrated by a high velocity projectile. Compos. Struct. 91, 103-109 (2009)

2. Wan, H., Bai, S., Li, S., Mo, J., Zhao, S., Song, Z.: Shielding performances of the designed hybrid laminates impacted by hypervelocity flyer. Mater. Des. 52, 422-428 (2013)

3. Larsson, F., Svensson, L.: Carbon, polyethylene and PBO hybrid fibre composites for structural lightweight armour. Compos. A: Appl. Sci. Manuf. 33, 221-231 (2002)

4. Chen, X., Zhou, Y., Wells, G.: Numerical and experimental investigations into ballistic performance of hybrid fabric panels. Compos. Part B 58, 35-42 (2014)

5. Del Rosso, S., Iannucci, L., Curtis, P.T.: Experimental investigation of the mechanical properties of dry microbraids and microbraid reinforced polymer composites. Compos. Struct. 125, 509-519 (2015)

6. Del Rosso, S., Iannucci, L., Curtis, P.T.: On the ballistic impact response of microbraid reinforced polymer composites. Compos. Struct. 137, 70-84 (2016)

7. Fujii, K.: Impact perforation behavior of CFRPs using high-velocity steel sphere. Int. J. Impact Eng. 27, 497508 (2002)

8. Sevkat, E.: Experimental and numerical approaches for estimating ballistic limit velocities of woven composite beams. Int. J. Impact Eng. 45, 16-27 (2012)

9. Cheeseman, B.A.: Ballistic impact into fabric and compliant composite laminates. Compos. Struct. 61, 161173 (2003)

10. Iremonger, M.J., Went, A.C.: Ballistic impact of fibre composite armours by fragment-simulating projectiles. Compos. Part A 26A, 575-581 (1996)

11. Prosser, R.A.: Penetration of nylon Ballistic Panels by Fragment-Simulating Projectiles. Part I: A linear approximation of the relationship between the square of the $\mathrm{V}_{50}$ or $\mathrm{Vc}$ striking velocity and the number of layers of cloth in the ballistic panel. Text. Res. J. 58(3), 161-165 (1988)

12. Prosser, R.A.: Penetration of nylon Ballistic Panels by Fragment-Simulating Projectiles. Part II: Mechanism of penetration. Text. Res. J. 58(3), 61-68 (1988)

13. Figucia, F., Williams, C., Kirkwood, B., Koza, W.: Mechanisms of improved ballistic fabric performance. (1982). Technical Report, U.S. Army Natick Research \& Development Center

14. Scott, B.R.:The penetration of compliant laminates by compact projectiles. In: Proceedings of the 18th International Symposium on Ballistics, pp. 1184-91. San Antonio, Texas (1999)

15. Lee, B. L., Walsh, T. F., Won, S. T., Patts, H. M., Song, J. W., Mayer, A. H.: Penetration failure mechanisms of armor-grade fiber composites under impact. J. Compos. Mater. 35(18) (2001)

16. Flanagan, M. P., Zikary, M. A., Wall, J.W., El-Shiekh, A.: An experimental investigation of high velocity impact and penetration failure modes in textile composites. J. Compos. Mater. 33(12) (1999)

17. Cunniff, P.M.: Dimensionless parameters for optimization of textile-based body armor systems. In: Proceedings of the 18th International Symposium on Ballistics, San Antonio, Texas, 1519 November 1999. p. $1303-10$

18. Ćwik, T.K., Iannucci, L., Curtis, P., Pope, D.:Project report: Ballistic performance of armox 370T and Armox 440T steels subjected to 20mm FSPs impact. Department of Aeronautics, Imperial College London. (2012). DOI: 10.13140/RG.2.1.4110.8643. Available at: https://www.researchgate.net/profile/TomaszCwik

19. Ćwik, T.K., Iannucci, L., Curtis, P., Pope, D.: Investigation of ballistic performance of Ultra High Molecular Weight Polyethylene composite panels. Compos. Struct. 149, 197-212 (2016)

20. STANAG 2920 Edition 2 Ballistic Test Method for personal armour materials and combat clothing (2003)

21. Ćwik T.K., Iannucci L., Curtis P., Pope D., Robinson P., Investigation of ballistic response of CFRP composites of various non-conventional reinforcement architectures. In: Proceedings of the 18th 
International Conference on Composite Materials. Jeju, South Korea, 28th August 2011. DOI: 10.13140 /RG.2.1.2630.1609. Available at: https://www.researchgate.net/profile/TomaszCwik

22. Hazell, P. J., Appleby-Thomas, G. J., Trinquant, X., Chapman D. J.: In-fiber shock propagation in Dyneema®. J. Appl. Phys. 110 (2011)

23. Mader, C.L. (Ed.): Los Alamos Shock Hugoniot Data. Los Alamos National Laboratory, (1980)

24. Millett, J.C.F., Bourne, N.K.: The shock induced equation of state of three simple polymers. J. Phys. D. Appl. Phys. 37, 2901 (2004)

25. Iannucci, L., Pope, D.: High velocity impact and armour design. Express Polym. Lett. 5(3), 262-272 (2011) 